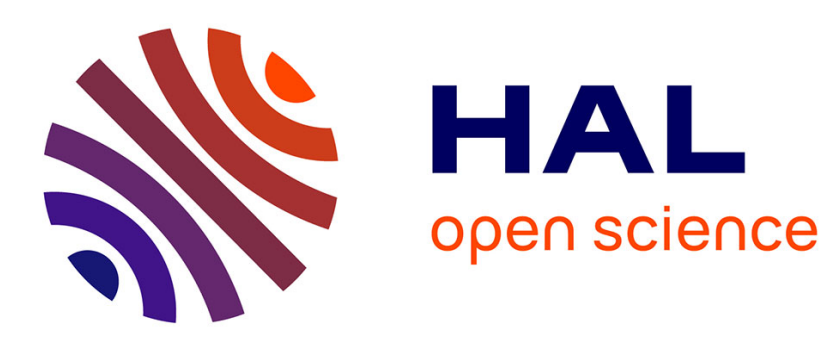

\title{
La surénonciation comme norme du genre: l'exemple de l'article de recherche et du dictionnaire en linguistique
} Francis Grossmann, Fanny Rinck

\section{To cite this version:}

Francis Grossmann, Fanny Rinck. La surénonciation comme norme du genre: l'exemple de l'article de recherche et du dictionnaire en linguistique. Langages, 2004, 38 (156), pp.34 - 50. 10.3406/lgge.2004.962 . hal-01737496

\section{HAL Id: hal-01737496 https://hal.science/hal-01737496}

Submitted on 19 Mar 2018

HAL is a multi-disciplinary open access archive for the deposit and dissemination of scientific research documents, whether they are published or not. The documents may come from teaching and research institutions in France or abroad, or from public or private research centers.
L'archive ouverte pluridisciplinaire HAL, est destinée au dépôt et à la diffusion de documents scientifiques de niveau recherche, publiés ou non, émanant des établissements d'enseignement et de recherche français ou étrangers, des laboratoires publics ou privés. 


\title{
La surénonciation comme norme du genre : l'exemple de l'article de recherche et du dictionnaire en linguistique
}

\author{
M. Francis Grossmann, MME Fanny Rinck
}

\begin{abstract}
Francis Grossmann, Fanny Rinck: Superenunciation as a norm of the genre: the example of linguistic research articles and dictionaries.

This article studies the posture of "superenunciator" in two genres that are both related to "enunciative effacement": French research articles and dictionaries in human sciences. According to our hypothesis, such a posture is required by these two genres, although it varies with their enunciative specificities. We first discuss how to analyse the plurality of enunciators and their relationships at the level of the text; this leads us to envisage a "polyphonic coherence" between the various viewpoints raised in a text. Then, by introducing the question of authorship in order to associate the enunciative responsibility with a textual one, we attempt to examine the role of discourse legitimization played by superenunciation. The analysis highlights some differences between the two genres: in dictionaries, the posture of superenunciator is related to the structural possibilities of the genre, and to a uniform enunciative authority. In the articles, it is directly construed by the plurality of enunciators, so that the author himself appears as a heterogeneous and divided figure.
\end{abstract}

\section{Citer ce document / Cite this document :}

Grossmann Francis, Rinck Fanny. La surénonciation comme norme du genre : l'exemple de l'article de recherche et du dictionnaire en linguistique. In: Langages, 38 année, $n^{\text { } 156, ~ 2004 . ~ E f f a c e m e n t ~ e ́ n o n c i a t i f ~ e t ~ d i s c o u r s ~ r a p p o r t e ́ s . ~ p p . ~ 34-50 ; ~}$

doi : $10.3406 /$ lgge.2004.962

http://www.persee.fr/doc/lgge_0458-726x_2004_num_38_156_962

Document généré le 01/06/2016 


\section{Francis Grossmann et Fanny Rinck}

Université Stendhal-Grenoble 3

Lidilem, E.A. 609

francis.grossmann@u-grenoble3.fr

fanny.rinck@u-grenoble3.fr

\section{La surénonciation comme norme du genre : l'exemple de l'article de recherche et du dictionnaire en linguistique}

\section{INTRODUCTION}

Si l'on se reporte aux définitions proposées par ses initiateurs (Rabatel, 2003), la surénonciation se définit par le fait qu'un énonciateur domine, au moins pour un temps, le jeu énonciatif. À l'oral, cette régie gouverne, entre autres, la gestion des tours de parole, la ratification ou l'invalidation du dit d'autrui, la reformulation. À l'écrit, la notion concerne la problématique du discours rapporté et plus largement la pluralité des voix dans le discours, que nous traiterons ici en termes de dialogisme ${ }^{1}$ en nous centrant sur la question de la hiérarchisation des énonciateurs.

Dans les genres écrits qui nous intéressent, la posture de surénonciation répond à une norme du genre : argumenter en faveur d'un point de vue oblige en effet à construire un texte qui marque la position de surplomb de l'instance de prise en charge énonciative. Est soulignée d'ailleurs, dans le cadre de la didactique de l'écrit (voir par ex. Boch \& Grossmann, 2002), l'importance de parler en son nom propre, pour éviter de produire un texte dans lequel se manifeste une position de sousénonciation.

Il existe différentes manières de se présenter comme surénonciateur et les genres actualisent différents procédés linguistiques permettant cette posture. Notre premier objectif est d'explorer cette diversité dans les deux genres étudiés (articles de recherche et dictionnaires de linguistique). On peut faire

\footnotetext{
1. Nous n'entrerons pas dans le débat terminologique concernant les distinctions entre les notions de "polyphonie " et de "dialogisme" (voir par ex. Bres et Verine, 2002, et Rabatel, dans l'introduction de ce numéro).
} 
l'hypothèse en effet que, tout en respectant les normes ${ }^{2}$ des genres académiques, ils produisent des formes spécifiques, liées à leur identité générique. Aussi, s'il est possible, empiriquement, de repérer les effets surénonciatifs associés à telle ou telle marque, ou plutôt à tel ou tel réseau de marques, il convient également de préciser la manière dont un genre de discours favorise potentiellement, à travers les caractéristiques discursives et linguistiques qui lui sont propres, la posture de surénonciation. Le deuxième objectif de notre analyse consiste à mettre en relation la prise en charge énonciative avec la responsabilité auctoriale et à montrer comment la surénonciation joue un rôle argumentatif ${ }^{3}$ de légitimation d'un point de vue d'auteur ${ }^{4}$.

\section{SURÉNONCIATION ET AUCTORIALITÉ}

\subsection{Cohérence polyphonique}

Une étude textuelle qui se donne comme objet d'analyser les effets de surénonciation en contexte d'effacement énonciatif, dans le cadre d'une approche attentive à la multiplicité des points de vue et à leur hiérarchisation, est loin d'aller de soi, puisqu'elle concerne différents niveaux d'analyse.

En son point de départ, notre approche conserve le cadre général de la théorie polyphonique développée par Ducrot. Le repérage des marques linguistiques, nécessaire à l'identification des instances de prise en charge énonciative (les énonciateurs) n'est cependant pas suffisant pour rendre compte de la prise en charge, au niveau textuel, des contenus assertés, lorsque le locuteur semble les prendre lui-même à son compte. Une telle prise en charge par L1 /E1 (selon la terminologie de Rabatel, dans l'introduction de ce numéro) implique qu'existe, en dépit de la prolifération des voix, ce que K. Flottum (2002: 342) propose de nommer une cohérence polyphoniqué, c'est-à-dire une cohérence entre les différents points de vue et leurs énonciateurs, cohérence dont on peut faire l'hypothèse avec cet auteur qu'elle est attendue par le récepteur.

2. Certaines formes de surénonciation, comme l'ironie par exemple, y sont sans doute tendanciellement prohibées, d'autres peuvent ètre fortement conventionnelles, jusqu'à être routinisées.

3. La notion d'argumentation est ici entendue au sens large, défini par J.B. Cirize (1990) en tant que démarche visant à modifier les représentations d'autrui, par les moyens du discours. Il existe a priori sur ce plan une différence entre les deux genres étudiés ici, puisque le statut du dictionnaire semble le conduire plutôt du côté de l'explication que de l'argumentation - le passage d'un destinataire profane à un destinataire d'experts conduisant inversement de l'explication à l'argumentation (P'lantin, 2002: 254). Malgré ces différences, nous postulons que les deux genres ont une visée argumentative de légitimation des points de vue. Nous revenons plus loin sur le statut de l'explication dans les deux genres.

4. Nous rejoignons ici, par une autre voie, la question des rituels sociolangagiers légitimant l'écriture, déjà abordée dans le $\mathrm{n}^{\circ} 105$ de la revue Langages (Beacco, dir., 1992), en y intégrant le problème de la responsabilité auctoriale. 
Une telle proposition revient à postuler que se joue, dans l'interprétation, un principe d'économie ${ }^{5}$ dans la prolifération des voix observée au niveau linguistique. Elle représente une solution intégrative, qui permet un regroupement - programmatique au moins - entre les différentes instances qui peuvent être indexées au locuteur, tout en les maintenant dans leur irréductible hétérogénéité ; ce point nous paraît essentiel car, on le verra pour le cas des articles de recherche, l'instance de prise en charge énonciative L1/E1 n'a rien d'homogène : pour le dire rapidement, on peut aussi bien avoir affaire par exemple au “je » de l'auteur empirique qu'à un énonciateur universel ${ }^{6}$.

La notion de cohérence polyphonique nous conduit alors à mettre en relation la dimension énonciative avec la problématique de l'auctorialité, i.e. cette instance extérieure au texte qui en assume la responsabilité : un point de vue repéré linguistiquement au niveau de l'énoncé ne peut en effet être indexé à L1/E1 que si l'on peut dire que ce dernier en est responsable, non en termes strictement énonciatifs mais en termes d'auctorialité. Au niveau énonciatif, l'ensemble des instances de prise en charge énonciative indexées à L1/E1 fonctionne donc comme une représentation dans le texte de l'instance auctoriale, responsable de la textualité et garante en cela des contenus assertés.

On rejoint ici ce que $\mathrm{M}$. Couturier (1995:22) traite en termes de figure de l'auteur, à avoir « l'auteur comme principal sujet énonciatif reconstruit dans l'acte même de lecture ». Désigner par le terme de "sujet » les instances énonciatives où s'originent les points de vue est cependant loin d'être satisfaisant, et il nous faut à nouveau insister sur l'hétérogénéité de l'énonciateur L1/E1 au niveau textuel. Notons que d'un point de vue sociolinguistique, la figure de l'auteur se dessine elle-même différemment, selon des variables caractérisant le type d'auteur que l'on prétend être ou que l'on est malgré soi, en particulier la discipline, le statut (auteur néophyte $v s$ auteur confirmé, maître ou disciple), la culture de laboratoire ou d'équipe ; elle prend aussi en compte les formes d'auctorialité (signature individuelle ou plurielle, construction éditoriale d'une hiérarchie des auteurs). On voit qu'une telle approche, si elle ne conduit pas à revenir sur la dissociation du couple position énonciative/statut ou place aboutit cependant à intégrer la variation sociohistorique dans l'analyse, au moins à titre de principe explicatif ${ }^{7}$, au même titre que la configuration opérée par les genres.

5. Voir la saturation des variables que les faits linguistiques ne peuvent saturer chez. K. Fløttum (ibid. : 341).

6. Ce problème est mis en évidence, en des termes proches, par M. Ali Bouacha (1995) qui, analysant comment se construit linguistiquement le sujet philosophique dans les Méditations de Descartes, montre la déformabilité des valeurs du "je ", dès lors que l'on dépasse le cadre phrastique pour observer son déploiement dans le texte, mais aussi parce que c'est une propriété de " je » dans ce discours que de transformer un marquage indiciel en catégorie philosophique.

7. Nous souscrivons au point de vue de Rabatel, qui refuse, à juste titre, toute interprétation mécanique de ces postures en termes de places, de rôles sociaux ou de statuts ; l'intégration de la variable sociale ne signifie pas qu'il y ait obligatoirement symétrie entre position énonciative d'une part, place ou statut d'autre part : le social et le langagier, bien que liés, restent deux ordres distincts. 


\subsection{Surénonciation et légitimation du point de vue d'auteur}

La notion de surénonciation ouvre des pistes intéressantes pour analyser la manière dont la dimension énonciative des textes joue un rôle argumentatif, dans le sens où la hiérarchisation énonciative favorise l'adhésion au point de vue de l'auteur. Le discours rapporté et le dialogisme, aux fondements de la posture de surplomb de L1/E1, peuvent en effet être envisagés comme participant d'une visée argumentative légitimant le discours, c'est-à-dire montrant sa qualité et sa pertinence scientifiques.

Certes, le discours rapporté et le dialogisme ont d'abord une fonction explicative dans les deux genres : les dictionnaires expliquent une notion en faisant référence aux travaux du domaine, cependant que, dans les articles, l'auteur doit montrer comment son approche se situe par rapport aux approches existantes. Explication et légitimation sont cependant indissociables : la construction des objets de connaissance ne va pas sans procédés par lesquels le discours se présente comme autorisé. La figure de garant qui se profile ainsi diffère suivant les genres : le dictionnaire légitime l'explication en la montrant comme scientifique mais aussi comme opératoire dans un champ donné, tandis que l'article met en évidence la scientificité et la singularité de l'approche présentée ${ }^{x}$. Une étude du dialogisme doit ainsi permettre de caractériser ces différences d'un point de vue interne, à travers l'établissement des relations hiérarchiques entre énonciateurs et de la cohérence des points de vue pris en charge par L1/E1.

\section{L'EFFACEMENT ÉNONCIATIF DANS LE “DISCOURS THÉORIQUE "}

Les genres discursifs en usage dans un domaine d'activité sont le produit des communautés de discours qu'ils contribuent à structurer (Swales, 1990) : de ce point de vue, les genres étudiés ont un statut institutionnel spécifique, et remplissent des fonctions différentes en termes d'apports au domaine disciplinaire. Si l'article concerne le versant de la production des savoirs, le dictionnaire s'attache à leur communication et leur diffusion. Cependant, comme on l'a souvent montré (voir par ex. D. Jacobi, 1999), il n'y a pas rupture mais continuum entre vulgarisation et recherche, ce qui peut expliquer aussi certaines caractéristiques communes. La dénomination discours théorique (DT) s'applique aux caractéristiques énonciatives des deux genres : les articles, comme les dictionnaires, représentent de manière relativement prototypique le DT, tel qu'il a été mis en évidence à partir des typologies textuelles fondées sur des critères énonciatifs (Benveniste, 1974, Simonin-Grumbach, 1975 et Bronckart, 1985). La spécificité du DT est qu'il tend à s'autonomiser par rapport à la situation d'énonciation, de sorte que l'interprétation peut se faire indépendamment

8. Il faudrait tenir compte également des différents types d'articles (enquêtes de terrain, réflexion épistémologique, synthèse théorique) : l'explication scientifique en dépend, et peut parfois se rapprocher plus ou moins de la didacticité caractéristique des dictionnaires. 
d'hypothèses sur la situation où l'énoncé a été produit. En d'autres termes, toute référence à la situation d'énonciation et toute trace de la subjectivité du locuteur y sont (tendanciellement) bloquées, suivant une procédure d'effacement énonciatif. Comme le montre G. Philippe (2002), signalant la nécessité de compléter l'approche proposée par Benveniste à propos de l'opposition discours vs récit, l'effacement énonciatif ne se caractérise pas uniquement en terme d'exclusions et d'absence, mais comme une possibilité qu'a le discours d'autonomiser son objet en semblant le couper du locuteur. Discours désembrayé et objectivant, le DT apparaît ainsi comme emblématique de ce qu'A. Rabatel (ici même) traite en termes d'effacement énonciatif maximal. Cependant, bien qu'heuristique, cette notion de DT ne doit pas masquer la diversité des genres concernés, leurs différences en termes de prise en charge énonciative, et de statut de l'auctorialité.

Nous appuierons notre réflexion sur une trentaine $d^{\prime}$ articles de revues ${ }^{9}$ concernant plus précisément la linguistique du discours et de l'énonciation, et sur quatre dictionnaires ${ }^{10}$, centrés également sur l'analyse du discours, la linguistique de l'énonciation ou la pragmatique. Notre hypothèse de travail part du fait que les articles de ces revues, comme les dictionnaires analysés, semblent reposer sur un même ethos (Maingueneau, 1984, p. 100), qui encourage la désinscription énonciative, tout en nécessitant cependant aussi, parallèlement, la construction d'un point de vue d'auteur. La désinscription énonciative qui les caractérise ne les conduit donc aucunement à les priver de locuteur, et joue elle-même un rôle dans la visée argumentative. Cette tension - qui varie évidemment en fonction des deux genres étudiés - conduit à poser en des termes spécifiques la question de la surénonciation. Comment, et sous quelles formes se construit la hiérarchisation des points de vue en faveur de L1/E1? Nous considérerons d'abord le cas des dictionnaires, dans lesquels la désinscription semble maximale, pour examiner ensuite les articles de revue, qui se révèlent, sous cet aspect, plus complexes.

\section{LA SURÉNONCIATION DANS LES DICTIONNAIRES}

\subsection{Logiques énonciatives du genre dictionnairique}

Relevons d'abord l'ambivalence du statut du dictionnaire en Sciences Humaines : en tant qu'outil didactique, il apparaît comme un discours second, par rapport aux discours scientifiques qui ont préalablement construit le

9. Les articles sont tirés de Cahiers de Praxématique (CDP), Faits de Langues (FDL), Langue Française (LFR), et Recherches linguistiques de Vincennes (RLV).

10. Les dictionnaires sont les suivants: Charaudeau, P. et Maingueneau, D. (dir.), (2002), Dictionnaire d'analyse du Discours, Paris, Seuil (désormais DAD) ; Détrie, C., Siblot, P., Verine, B. (dir.) (2001), Terme's et concepts pour l'analyse du discours, une approche praxématique, Paris, Champion (désormais TCAD); Moeschler, J. et Reboul, A. (1994); Dictionnaire encyclopédiqué de pragmatique, Paris, Seuil (désormais DEP); de Nuchèze, V. et Colletta, J.-M. (dir.) (2002), Guide terminologique' pour l'analysé du discours, Berne, P. Lang (désormais GTAD). 
champ ; mais, en tant qu'instrument de légitimation, il peut être considéré aussi comme un genre constituant (Maingueneau et Cossutta, 1995) servant de garant à une communauté, ici disciplinaire, ou encore de manifeste d'école. Cette dualité a son corrélat énonciatif : puisque la logique du dictionnaire repose d'abord sur la définition des termes, la question reste parfois ouverte de savoir si les normes définitionnelles sont celles des auteurs du dictionnaire ou bien si elles appartiennent au cadre théorique auquel se réfère la définition. Ainsi s'explique l'ambiguité de certaines définitions, comme celle-ci :

(1) L'intervention ne doit pas être confondue avec le tour de parole (TCAD, “Intervention", p. 159).

Convient-il ici d'analyser la prescription comme émanant des auteurs de la terminologie, ou bien doit-on admettre que les auteurs du dictionnaire en endossent aussi la responsabilité ? On voit les ambiguités de la doxa : dans les cas de quasi fusion des points de vue, l'énonciateur confère bien un statut universel au discours, son propre point de vue se fondant dans celui d'autrui. Dans d'autres, comme celui qui vient d'être cité, qui concerne surtout les termes les plus techniques, L1/E1, s'il continue à offrir sa caution, la restreint au cadre dont elle est issue. À l'effacement énonciatif, constitutif du genre, correspond alors un effacement argumentatif. On peut parler de sousénonciation, l'énonciateur - dans l'article concerné - jouant le rôle de simple relais. Dans d'autres cas, au contraire, l'effacement apparent se double d'une mise en perspective et d'une discussion du point de vue rapporté, en fonction de celui qu'assume l'auteur de l'article, ou de la ligne éditoriale de l'ouvrage : l'appartenance à une école particulière (la praxématique, dans le cas du TCAD), l'adhésion à un point de vue (la théorie de la pertinence de Sperber et Wilson dans le DEI') ou bien encore le prisme disciplinaire adopté (l'analyse du discours pour le IDAD, la pragmatique interactionnelle pour le GTAD). Un point de vue d'auteur se manifeste à deux niveaux, au sein de l'article même, mais aussi à travers l'ouvrage dans son ensemble, qui semble dessiner la figure d'un superénonciateur transcendant les points de vue particuliers. Cependant, l'idée qu'un énonciateur englobant et unique affleurerait en permanence rendrait mal compte de la diversité observée : dans le genre dictionnairique, fondé sur la division en notions ", la cohérence polyphonique, si elle n'est pas exclue, reste toujours problématique. À l'hétérogénéité des référents théoriques s'ajoute le statut complexe de l'auctorialité, en raison du caractère collectif des ouvrages, et de l'inégalité de statut des différents auteurs. Cette diversité est masquée par l'uniformité apparente du système énonciatif, l'utilisation quasi exclusive du délocuté étant la norme et la valeur du on étant plutôt celle d'un indéfini universalisant, le nous étant généralement exclu ${ }^{12}$.

11. Avec des différences notables suivant les dictionnaires : ainsi le DEP maintient un découpage en chapitres, permettant également une présentation beaucoup plus développée des notions.

12. Même si l'on trouve quelques rares dérogations à ce principe. 


\subsection{Des formes de surénonciation inscrites dans l'organisation structurale}

En sélectionnant les entrées, le dictionnaire intronise les notions admises, le système des renvois spécifiant et limitant les parcours conceptuels possibles. Cette sélection permet aussi de distinguer deux instances auctoriales distinctes quoique complémentaires. La première conduit à officialiser les notions dans le champ disciplinaire ${ }^{13}$, en leur conférant la dignité de notions clés, puisque répertoriées. La seconde constitue chaque auteur d'article comme responsable de son énonciation : les notions appartenant en propre aux auteurs, principaux ou secondaires, du dictionnaire (souvent développées par ces auteurs euxmêmes, dans le DAD) font l'objet d'une mise au point dans laquelle un point de vue critique peut être exprimé.

Le procédé du renvoi, grâce à la mise en gras et à l'astérisque, offre un moyen métalinguistique proprement scriptural de surplomber un point de vue, comme le montre l'exemple (2) : récusant l'approche de la langue comme système, l'article enserre l'approche systémique de la langue dans les mailles d'un filet conceptuel de renvois, qui conduisent à démonter la notion même qui est analysée :

(2) L'éviction des usagers de la parole au profit du système institué a pour conséquence la réification* du sens et la non-prise en compte de la production de sens. Les interactions verbales*, les ratages de la parole* ne peuvent y prendre place. (TCAD, "Système ", p. 337).

La notion saussurienne de système se trouve ainsi condamnée, non seulement par le fait qu'elle tend à "réifier » la langue, mais aussi par tout ce qu'elle laisse échapper (et qui fait l'objet d'entrées spécifiques du dictionnaire).

\subsection{L'énonciateur universel comme surénonciateur}

Le dictionnaire, on l'a vu, tend à effacer le dialogisme pour présenter un point de vue doxique. Pour ce faire, il met en scène de manière privilégiée un énonciateur universel ${ }^{14}$, dont les garants sont variables : il peut renvoyer à la Science (le dictionnaire entérine ce qu'il évalue comme validé scientifiquement), comme à l'usage (le dictionnaire signale la prédominance d'un point de vue ou d'une définition dans le champ) ${ }^{15}$. Les dictionnaires pratiquent peu le discours rapporté, ils utilisent plutôt un discours multiréférencé, dans lequel les noms propres d'auteurs jouent le rôle de balises théoriques (Grossmann, 2002,

13. D'une manière générale, dans le DAD, l'cecuménisme affiché permet à nombre d'auteurs de présenter "leur» notion, tandis que dans le TCAD, le souci de cohérence d'école fait dominer la logique collective.

14. La manière dont est mis en scène cet énonciateur universel varie en fonction de la conception adoptée (par ex. dictionnaire d'école vs dictionnaire de synthèse).

15. Par exemple, à l'entrée 'Intertextualité du DAD, p. 328 : "L'usage a tendance à employer intertexte quand il s'agit de relations à des textes sources précis (citation, parodie) et interdiscours pour des ensembles plus diffus : ainsi on dira plutôt "La parole s'exerce dans un vaste interdiscours" ". 
2003) et confortent le point de vue de L1/E1. Ce phénomène est renforcé par le recours aux formes de l'énonciation historique qui permet, pour plagier le mot de Benveniste, de laisser les théories parler elles-mêmes, en refusant toute subjectivité apparente. L'instance évaluative - ou sujet modal - se manifeste sous des formes bien connues (notamment à travers la modalisation appréciative, ou la présupposition: Ce principe est censé "remplacer" les maximes conversationnelles, GTAD, "Inférence », p. 84). La prise en charge d'un point de vue critique est en outre souvent euphémisée, par des modalités du type sembler, ponvoir ou par le recours à un on qui joue sur l'indistinction de ses valeurs exclusives et inclusives:

(3) On peut aussi reprocher aux analyses se voulant strictement linguistiques d'avoir répandu l'idée que la négation n'est complète que lorsqu'elle affecte le prédicat : l'influence de la conception logique de la négation y est certainement pour beaucoup. (TCAD, « Négation », p. 199).

Paradoxalement, cette euphémisation même conduit à une forme de surénonciation : en tant que discours doxique, le dictionnaire, lorsqu'il exprime un point de vue, cherche à l'exprimer au nom d'une vérité universelle et la forme euphémisée liée à l'ethos fournit une garantie supplémentaire de scientificité.

Le dictionnaire évalue en outre la pertinence des notions traitées en entrées en les plaçant dans un cadre historique ou conceptuel. Le cadrage ${ }^{16}$ historique a pour fonction de situer une notion en la rapportant à son origine, et conduit parfois aussi à questionner son statut disciplinaire (On peut s'interroger sur le bien-fondé de céte' entrée, TCAD, "Idéologie", p. 146). Il se marque dans l'incipit des articles des dictionnaires, qui comprend souvent les adverbes traditionnellement ou classiqueme'nt... venant modifier des verbes de dire, de nomination ou de définition avec le on indéfini (on dit qué, on appelle, on définit...). D'autres structures jouent le même rôle : des passifs à valeur impersonnelle (traditionnell'me'nt, à l'origine'... e'st appelé $X)$ ou encore des formules métalinguistiques faisant du nom défini l'agent de sa propre définition ( $X$ dénomme traditionnellement, habituelleme'nt..., $X$ renvoie traditionnellement à...).

Le cadrage conceptuel s'effectue à partir de la présentation préalable d'un discours appartenant au sens commun ou à l'intuition, auquel le dictionnaire oppose ensuite un point de vue se voulant plus exact. La posture surénonciative impliquée par cette forme de cadrage se manifeste aussi à travers la manière de découper le champ disciplinaire, y compris à travers la détermination des paradigmes, comme le montre le découpage binaire opéré dans le DEP :

\footnotetext{
À l'heure actuelle, il existe deux paradigmes principaux en pragmatique, que nous nommerons pragmatique intégrée et pragmatique cognitive (...). (DEP', p. 79).
}

16. Nous appelons ici cadrage le procédé qui consiste à contextualiser historiquement lés notions définies, mais aussi à spécifier leurs principales oscillations conceptuelles dans le champ. 
Ce privilège de la nomination des paradigmes résulte de la fonction même du dictionnaire : s'il rappelle l'usage, il institue aussi une cartographie conceptuelle.

\section{4. Énonciation historique et narrativisation}

Le genre dictionnairique résout les différences d'approche à travers une vision téléologique, qui programme le déroulement des évolutions. Le surplomb théorique, impliquant une mise en scène narrative des points de vue, se marque typiquement à travers des déictiques textuels tels que désormais ou depuis, des particules négatives impliquant l'impossibilité du retour en arrière comme ne... plus, des conjonctives comparant un état antérieur et un état actuel ou encore des adverbes indiquant la préférence comme plutôt ; le point de vue dominé est rejeté dans l'arrière-cour de l'histoire, ce dont témoignent aussi, comme dans l'exemple (5), les antithèses lexicales (significations antagonistes vs significations multiples et mouvantes) :

(5) une partie des lexicologues prennent désormais leurs références philosophiques chez C.S. Peirce ou chez L. Wittgenstein (" faites-vous enseigner la signification par l'usage ", $1986: 235)$ plutôt que chez F. de Saussure. En analyse du discours - pour le courant le plus proche des ethnologues - l'unicité du signe lexical n'est plus postulée ; le sens se construit dans l'interaction et imbrique le mot dans les activités pratiques d'acteurs* situées dans des contextes d'action variés. Les significations ne sont pas davantage " antagonistes » comme lorsque $M$. Pêcheux travaillait sur des discours politiques, elles apparaissent plutôt multiples et mouvantes »(DAD, « Mot », p. 396).

La visée argumentative s'inscrit au cœur du dispositif de narration, l'énonciation de type historique tendant à transformer les notions elles-mêmes en actants du récit scientifique : les notions s'autonomisent, et fonctionnent comme agents, l'introduction d'un point de vue d'auteur se faisant sous couvert des propriétés de la notion elle-même :

(6) La notion d'interaction verbale vient corriger le schéma de la communication popularisé par Jakobson (1963) (...) (TCAD, "Interaction verbale », pp. 152-153).

En présentant les paradigmes sur le mode narratif, l'énonciateur principal, qui connaît le sens de l'histoire, qualifie ou disqualifie les théories et les points de vue : ainsi, telle thèse $a$ depuis été battue en brèche par la praxématique (TCAD, "Langue/Parole/Discours», p. 169), ou doit laisser la place (DAD, "Conditions de production » p. 119). L'argumentation recourt au lexique pour marquer la domination du point de vue et s'appuie aussi sur des rôles pré-définis, à l'aide d'étiquettes généralisantes (en gras dans l'exemple 7). Ainsi, plutôt que de défendre directement une thèse ou une assertion, les auteurs du dictionnaire attribuent des rôles qu'ils font jouer aux acteurs du champ, et se placent en position d'arbitres, grâce à des procédés bien connus (soulignés dans l'exemple) : modalisateurs appréciatifs, adverbes paradigmatisants (Nølke, 1983) marquant la focalisation (du type surtout ou même), focalisateurs à valeur contrastive ( $c^{\prime}$ est au contraire...), etc.

(7) Les pragmaticiens ont parfois reproché aux spécialistes de l'énonciation de s'intéresser exclusivement aux relations énonciateur-énoncé, et de ne pas 
tenir compte, dans leurs analyses, des fonctionnements et contraintes de l'interlocution. C'est surtout vrai des travaux relatifs à la subjectivité langagière (...), dont le fondement paraît être le présupposé subjectiviste selon lequel le sujet-locuteur est seul maître de son discours. Dans les travaux portant sur la polyphonie et l'intertextualité, c'est au contraire l'intersubjectivité qui est mise au premier plan. (GTAD, "Énonciation ", p. 65).

à partir des deux points de vue mis en scène, celui des «pragmaticiens " et celui des "spécialistes de l'énonciation"; il n'y a pas effacement énonciatif de la voix de E1, mais construction d'une position en retrait, qu'on peut qualifier ici aussi de surplombante (elle produit un effet de surénonciation) ${ }^{17}$. $D^{\prime}$ une manière générale, le recours à ces étiquettes permet de renforcer une vision scénique du champ scientifique, en réifiant les courants ou les problématiques, présentés plus ou moins positivement par l'énonciateur principal, avec un effet de synthèse classante:

(8) Les problématiques du dialogisme* ou de l'hétérogénéité constitutive montrent que l'intradiscours est traversé par l'interdiscours. (DAD, «Intradiscours ", p. 329).

\subsection{Surénonciation et aspects dialogiques de l'argumentation}

Une présentation apparemment dialogique peut valoriser un point de vue, dont la domination se construit en creux, à partir d'une critique raisonnée des fondements d'une notion princeps (cf. l'article «Motivation », dans le TCAD, pp. 194-195). Mais en général, les dictionnaires s'efforcent plutôt de présenter une vue panoramique, en identifiant les courants ou les noms d'auteurs, considérés comme autant d'angles de vue possibles sur la notion à définir. Les expressions linguistiques qui permettent de le faire sont des cadreurs (Wilmet, 1998 : 538) comme Pour [Nom de discipline, d'auteur ou d'école], ou Du point de 7u' de [Nom de discipline ou d'écolel dont l'usage se révèle cependant ambivalent. Ces cadreurs permettent en effet d'introduire une succession de points de vue, dont l'égale dignité est reconnue. Mais ils offent aussi le moyen, lorsqu'ils apparaissent en synthèse, de marquer la domination d'un point de vue particulier. Les cadreurs pour ou du point de vue de introduisent alors un développement venant résoudre des difficultés mises en évidence dans l'article ${ }^{18}$. Plus subtilement, ils reformulent, en fonction d'un nouveau cadre conceptuel, la définition d'une catégorie ancienne, comme ci-dessous en (9) à propos des figures rhétoriques:

(9) Iour la praxématique, la figure manifeste exemplairement la dialectique de la production de sens, effectuée dans le temps de l’à-dire*. (...) (TCAD, "Figure ", p. 125).

17. Les modaux de désinscription énonciative (cf. "dont le fondement paraît être ») ont moins pour fonction d'atténuer la portée polémique du propos (cf. « le présupposé subjectiviste ») que de porter l'ethos objectif convenant au genre scientifique.

18. À la différence de selon, qui n'est pas utilisé pour marquer le point de vue dominant dans la synthèse conclusive. 
La question du statut de l'auteur interfère aussi dans cette problématique dialogique. Dans le TCAD, dictionnaire d'école, la fusion est constante, et d'ailleurs pleinement assumée, entre le point de vue de l'énonciateur principal et le point de vue de l'école praxématique. La figure de l'auteur est théoriquement pré-construite, le travail opéré consistant à évaluer les concepts et les théories présentés à l'aune de cette approche. Dans les autres dictionnaires, à l'exception peut-être du DEP, qui se veut moins un dictionnaire qu'une synthèse critique, la construction d'une figure d'auteur collectif reste plus incertaine, et mouvante. Dans le $\mathrm{DAD}$, par exemple, où l'on a affaire à deux auteurs principaux, le principe de juxtaposition est parfois utilisé, entérinant l'égalité de traitement énonciatif :

(10) La notion de compétence discursive prend des valeurs variables selon le sens qui est donné à "discursive ", elle est souvent mise en contraste avec la notion de "compétence linguistique » introduite par N. Chomsky. (...)

Pour P. Charaudeau (2000b), (..);

Chez D. Maingueneau (1984), (DAD, "Compétence discursive », pp. 113-114).

La présentation des points de vue dans les dictionnaires n'aboutit pas toujours à mettre les énonciateurs mis en scène sur un pied d'égalité. L1/E1 peut par exemple exprimer son accord ou son désaccord avec e2, en situant le point de vue par rapport au cadre d'analyse qu'il privilégie :

(11) Une telle modélisation entre en cohérence avec les hypothèses fondatrices de la praxématique, qui place au centre de sa théorisation le sujet producteur de discours et la construction des sujets en discours. Elle peut servir de cadre englobant aux études linguistiques de Bres (1993) sur la production d'identité sociale dans le récit oral, ainsi qu'aux analyses textuelles réunies par Bres, Détrie et Siblot (1996) sur les figures de l'interculturalité* dans le roman contemporain. (TCAD, "Ipséité », p. 162).

Mais il peut s'appuyer également sur un énonciateur secondaire utilisé pour disqualifier un point de vue ou une approche. L1/E1 fait ainsi endosser à e2 la responsabilité d'un point de vue critique (cf. la fin de l'article "Méthode harrissienne " du DAD, p. 379); on trouve là une forme de sousénonciation qui n'est que provisoire, l'ethos "académique " permettant souvent à L1/E1 de reprendre la main in fine, en s'appuyant sur des structures concessives (cependant, néanmoins) ou sur des conditionnels. Le dictionnaire - en particulier le DAD - adopte en effet un ton consensuel, visant à valoriser les apports, même lorsqu'ils sont issus d'une théorie présentée de manière critique.

\section{LA SURÉNONCIATION DANS LES ARTICLES DE REVUE}

\subsection{Des coups de force dans des énoncés impersonnels}

Quel que soit leur objet, les articles de revue développent toujours une argumentation en faveur du point de vue de l'auteur ${ }^{14}$, qui vise à y présenter et promouvoir sa recherche.

19. Y compris dans les cas de signatures plurielles, la norme d'usage veut que ce point de vue soit unifié. 
Le point de vue de L1/E1 peut être exprimé par une énonciation personnelle : les objectifs, choix terminologiques et méthodologiques sont souvent précisés au moyen de nous, voire $j e$, rattachés à des verbes métadiscursifs.

Mais en vertu de l'objectivation caractéristique du discours scientifique, ce point de vue se construit principalement dans une énonciation au présent, où prime le délocuté, avec le recours à des tours impersonnels ainsi qu'à un on à valeur universalisante et indéfinie, englobant et dépassant les références personnelles au locuteur et à l'interlocuteur (on le sait, on comprend mieux pourquoi).

Si les contenus sont ainsi pris en charge par un énonciateur universalisant, le sujet modal se manifeste dans des énoncés impersonnels par des coups de force assertifs, comme l'utilisation de la modalité déontique dans des tours caractéristiques de la phraséologie de l'article (il faut reconnaitre, on doit, force est de...).

Observons l'exemple (12):

(12) Chacun à leur manière, tous ces exemples militent en faveur d'une approche unifiée et homogène des citations directes et indirectes d'une part, libres ou contraintes d'autre part (FDI, 19, p. 157).

Alors que l'approche en question a été introduite au début de l'article comme un choix théorique de l'auteur (nous voudrions montrer, nous soutiendrons), cette phrase inaugurant la conclusion apparaît comme un coup de force de l'assertion : par la modalisation que porte le verbe, et le statut syntactico-sémantique des exemples, ceux-ci sont présentés comme responsables d'une action de militantisme. L'expression, quasi lexicalisée, fait apparaître une forme de surénonciation routinisée, induite par la phraséologie même. Rapporté à l'économie du texte, cet énoncé est assé représentatif du fonctionnement énonciatif de l'article, qui se caractérise par un transfert de l'instance énonciative : explicitement mis en scène comme responsable de la textualité et de la conceptualisation qui s'y élabore, L1/E1 disparaît selon un procédé de désinscription énonciative, l'argument apparaissant alors comme indépendant de tout locuteur.

Ce parcours énonciatif montre qu'on n'a pas d'un côté un énonciateur universalisant, et d'un autre, surgissant sporadiquement, une présence auctoriale qui s'exprimerait de manière univoque par une énonciation personnelle: c'est par la tension entre effacement énonciatif et immixtion du sujet modal que se définit la prise en charge énonciative dans l'article. En permettant le passage et la dilution de la frontière entre énonciation personnelle et universelle, l'usage ambivalent de on traduit cette tension, par laquelle le point de vue de L1/E1 se positionne comme point de vue surplombant et tire son autorité, comme le montre également le dialogisme dans:

(13) Ce n'est évidemment pas une redécouverte du personnage classique, du héros réaliste ; au contraire, ce qui se profile ici, c'est un sujet frappé d'indistinction (...) (RLV 28, p. 36).

L'opposition à un point de vue implicite, doublement marquée par la négation et le connecteur au contraire, et appuyée par la focalisation, sert l'argumentation en faveur du point de vue de L1/E1, l'adverbe évidemment venant encore renforcer l'autorité que ce point de vue doit à sa position de surplomb. 


\subsection{Modes d'insertion et évaluation des autres points de vue attestés dans le champ disciplinaire}

La posture de surénonciation tient à la logique énonciative du genre de l'article et de sa phraséologie : elle résulte notamment des marques dialogiques du type de celles que nous venons d'évoquer, où un point de vue implicite permet à L1/E1 de faire valoir le sien; c'est aussi l'effet que produit la mise en scène d'autres points de vue présentés comme des points de vue attestés dans le champ.

Il importe en effet pour l'auteur de l'article de situer son approche par rapport à d'autres; s'il doit pour ce faire s'y référer explicitement, le renvoi à d'autres travaux ne fonctionne pas toujours sur le mode de la transparence : il peut emprunter les voies de l'évocation voire de l'allusion (Boch, Grossmann, 2002 ; Grossmann, 2002). Dans tous les cas cependant, et bien qu'il soit possible pour L1/E1 de procéder momentanément à un simple état des lieux qui le conduit provisoirement à s'effacer, la mention d'autres approches s'inscrit toujours dans la visée argumentative $\mathrm{du}$ texte, de sorte que le point de vue de l'auteur se présente dans une position de surplomb.

\subsubsection{Un énonciateur autonome}

Si des ambiguïtés énonciatives demeurent, un ensemble de procédés de distanciation sont mis en ceuvre dans le texte pour que la voix de l'auteur soit clairement distinguée de celle d'autrui. Cela peut aller jusqu'à nier le rôle de coconstructeur des savoirs de $12 / \mathrm{e} 2$, dans les cas où la référence à $12 / \mathrm{e} 2$, postposée au contenu propositionnel, est introduite par une formule comme voir aussi : L1/E1 se présente ainsi comme seul responsable des propos; signalant toutefois qu'ils ont été produits aussi par d'autres, l'auteur se montre en cela fidèle à la déontologie scientifique, et retire de cette stratégie le double bénéfice de la légitimité du dit, et de son attribution.

\subsubsection{Effacement de la source du point de vue}

La mention de points de vue attestés dans le champ n'implique pas nécessairement, on l'a dit, une identification exhaustive de leur source énonciative. Il existe en effet nombre d'identifications sans nom d'auteur, qui peuvent prendre des formes différentes. Il peut s'agir d'étiquettes généralisant des positions du champ (les stylisticiens, les nominalistes, les détracteurs de [telle conception]), de désignations hyperonymiques (les auteurs), ou d'identifications indéterminées (certains, d'aucuns, on), voire implicites, seul un verbe épistémique en construction passive ou infinitivale étant représenté (par ex. dans analyser $X$ comme... revient à...).

Tout comme dans les dictionnaires, L1 /E1 s'octroie, par les étiquettes généralisantes, le privilège de la nomination des paradigmes ; ce procédé peut par ailleurs s'accompagner d'une position d'arbitrage, L1/E1 s'attribuant le rôle de tiers en proposant une alternative nouvelle dans un débat. Les autres formes $d^{\prime}$ identification sans nom d'auteur fonctionnent sur un principe d'indétermination de la source énonciative, et produisent des effets de surénonciation: 
de prédication seconde, sont souvent traitées comme (...). Une approche quelque peu normative tente de justifier le fonctionnement de ces groupes (...) (LF 135, p. 100).

On observe une dépréciation des approches ainsi désignées, et une connivence avec un lecteur idéalement positionné comme maîtrisant suffisamment le champ pour être apte à décoder l'implicite.

\subsection{3. Évaluation des points de vue attestés dans le champ}

Parfois, les thèses sont mentionnées sans que L1/E1 donne explicitement son point de vue, leur recontextualisation et leur agencement textuel servant l'enchaînement argumentatif ${ }^{20}$. Mais, le plus souvent, les points de vue d'autres auteurs font l'objet d'évaluations positives ou négatives de la part de L1/E1, qui signale ainsi son accord ou son désaccord.

(15) Dans son étude, il écrit que [citation]. L'excellente description que cet auteur donne des emplois de ko (...) (FDL 19, p. 70).

L'axiologique positif, conjoint à l'effacement énonciatif, place L1/E1 dans la position dominante de celui qui juge de la qualité scientifique des travaux de ses pairs.

De manière générale, l'évaluation, qu'elle soit positive ou négative et concerne un point de vue pris en charge ou non par L1/E1, se fait sur la base de critères de scientificité : la précision, la clarté, la profondeur d'analyse - opposée au sens commun - mais aussi la nouveauté - par rapport à ce qui est qualifié $\mathrm{d}^{\prime}$ ancién ou de traditionnel - en sont les paradigmes privilégiés. On trouve ici une caractéristique commune aux deux genres. Parce qu'elle emprunte en outre des tours objectivants tout en se présentant comme modérée, l'évaluation dans l'article confère à L1/E1 les propriétés de l'ethos scientifique, orientant ainsi le lecteur dans le sens escompté par l'auteur.

\subsection{Un surénonciateur décentré}

Le point de vue de L1/E1 se trouve valorisé par la hiérarchisation énonciative, qui sert l'argumentation. Mais la cohérence textuelle et conceptuelle ne doit pas masquer le fait que L1/E1, loin d'être une instance homogène et unifiée, se présente au contraire comme une instance décentrée, qui adopte provisoirement certaines positions antagonistes:

(16) Dans ces conditions, ne ferait-on pas mieux de reconnaître que ce que nous nous obstinons à concevoir comme une forme de démonstration, c'est-à-dire de citation, portant sur le contenu d'un discours objet, n'est en fait qu'une simple extension de ce qui est décrit par le locuteur? (FDL 19, p. 154)

selon une stratégie de reconstruction du processus de la découverte qui manifeste un effet de surénonciation renforcé par la question rhétorique. Plus largement, L1/E1 revient sur ses propres assertions pour les commenter, adoptant

20. Dans les cas de désaccord par exemple, le point de vue peut être disqualifié simplement par la négation ou par des connecteurs d'opposition. 
ainsi une attitude métadiscursive, comme le montre le dédoublement énonciatif entre le corps du texte et les notes de bas de page ci-dessous :

(17) Le fait qu'immanquablement ", pour les items lexicaux, la dénomination débouche sur une relation de sens codée permettant l'utilisation de la dénomination pour les occurrences du concept général dénommé nous a conduit in fine à poser le caractère sémantique codé comme trait définitoire des dénominations métalinguistiques : il y a dénomination, parce que l'item lexical a un sens codé ou préconstruit.

[En note : ] a. Le immanquablement est bien sûr trop fort, puisque l'on peut fort bien, pour des items lexicaux comme or, eau, chat, etc., renoncer à un sens descriptif ou représentationnel et adopter, précisément à cause de leur vicinité dénominative, le mode de fonctionnement causaliste (la référence causale) postulé pour les noms propres par Kripke (1972) et Devitt (1974 et 1976). (CDP 36, p. 27.)

Le jeu complexe des instances énonciatives, au niveau de la note et du dédoublement qu'elle introduit - c'est aussi un jeu avec les normes du genre - pose la question de leurs rapports : surénonciation et sousénonciation s'entremêlent, la note invalidant le dit, mais fonctionnant aussi comme forme de garantie de l'objectivité de l'assertion.

Décider dans ce cas précis du rattachement des instances énonciatives à L1/ E1 suppose donc de concevoir plus largement la figure de l'auteur dans l'article comme une instance clivée qui va jusqu'à assumer ses diverses facettes et même ses contradictions. Simulant la découverte, imposant son point de vue sur le monde, et, socialement, son identité de chercheur, il est ce surénonciateur multiple qui se présente à la fois comme le responsable du texte, de la recherche qui y est présentée, et comme la science elle-même, ou son représentant.

\section{CONCLUSION}

L'effacement énonciatif propre au discours théorique semble bien jouer, dans les deux genres, un rôle de surénonciation. Dans les dictionnaires comme dans les articles, la construction d'un point de vue d'auteur s'effectue à travers l'immixtion du sujet modal, sous le couvert de l'énonciateur universel. Dans les dictionnaires, si les rôles alternent (didactique, épistémologique, critique...), du point de vue énonciatif, ils sont subsumés par une instance qui se présente comme homogène et englobante. Les effets de surénonciation s'appuient alors sur les possibilités offertes par le dictionnaire comme méta-discours : possibilité de sélectionner les entrées, de distinguer différents points de vue sans les intégrer obligatoirement ou à l'inverse, de présenter un point de vue qui domine structurellement les autres. Dans l'article, le surénonciateur se constitue par transferts de l'instance énonciative, hétérogène et mise en scène jusque dans ses contradictions. L'effet de connivence apparaît davantage, notamment dans les références à d'autres auteurs. La visée argumentative prend donc des formes différentes dans les deux genres: dans le dictionnaire, elle semble s'effacer pour mieux atteindre son but, grâce au style assertif de l'énonciation historique. Dans l'article scientifique, elle repose directement sur la pluralité des énoncia- 
teurs, et sur la figure d'un auteur qui, tout en se présentant comme une caution de scientificité, instanciable en tant que telle à l'envi, n'a de cesse d'affirmer sa singularité.

Terminons par une interrogation : a-t-on vraiment besoin de la notion de surénonciation? Prise en elle-même, elle n'offre pas de prise originale sur le fonctionnement de phénomènes énonciatifs isolés, déjà bien étudiés. Son intérêt est qu'en rendant plus lisible le lien qui peut exister entre effacement énonciatif et visée argumentative, elle met en évidence les effets pragmatiques de la multiplicité et de la hiérarchisation des instances énonciatives; l'ensemble des procédés linguistico-discursifs mis en ceuvre pour poser L1/E1 en figure d'auteur surplombante ravale en effet de facto le point de vue des énonciateurs secondaires. Le préfixe sur ne doit pas cependant faire illusion : il ne représente aucunement la maîtrise par le sujet de son discours, mais traduit plutôt le fait que l'argumentation, inscrite dans la langue, s'inscrit tout aussi naturellement (c'est-à-dire quoi qu'on fasse) dans le discours, et dans les genres qui le constituent. Les configurations opérées par les genres ne prédéterminent pas entièrement l'usage singulier que peuvent en faire les scripteurs. En se constituant comme auteurs, ils peuvent exploiter les règles du genre - voire s'en jouer mais sont aussi joués par lui.

\section{Références bibliographiques}

Alı Bouacha Magid, 1995, "De l'ego à la classe de locuteurs : lecture linguistique des Méditations ", Langages 119, 79-94.

BEACCo Jean-Claude (dir.), 1992, Ethnolinguistique de l'écrit, Langages, 105.

Benveniste Émile, 1966/1974, Problèmes de linguistique générale, t. I et Il. Gallimard, Paris.

Boch Françoise, Grossmann Francis, 2002, "Se référer au discours d'autrui, quelques éléments de comparaison entre experts et néophytes ", Enjeux 54, 41-51.

BRES Jacques, VERINE Bertrand, 2002, "Le bruissement des voix dans le discours: dialogisme et discours rapporté ". Faits de langues 19, 159-169.

BRONCKART Jean-Paul, dir., 1985, Le fonctionnement des discours, Delachaux et Niestlé, Paris.

Couturier Maurice, 1995, La Figure de l'auteur, Seuil, Paris.

FLøTTUM Kjersti, 2002, "Polyphonie au niveau textuel ", Romansk Forum 16, 339-350, http:/ /www.digbib.uio.no/roman/Art/Rf-16-02-2/fra/Flottum.pdf.

GRIZE Jean-Blaize, 1990, Logique et langage, Ophrys, Gap, Paris.

GrosSMANN Francis, 2002, "Les modes de référence à autrui : l'exemple de la revue 'Langages' ", Faits de langues 19, 255-262.

GRosSmanN Francis, 2003, "Du discours rapporté au discours autorisé, le maniement des noms d'auteur dans l'article en Sciences Humaines ", Estudios de Lengua y Literatura francesas 14, 9-26.

JACOBI Daniel, 1999, La communication scientifique : discours, figures, modèles, Presses Universitaires de Grenoble, Grenoble.

MAINGUENEAU Dominique, CossutTA Frédéric, 1995, "L'Analyse des discours constituants ", Langages 117, 112-125.

MoesCHLER Jacques, Reboul Anne, 1994, Dictionnaire encyclopédique de pragmatique, Paris, Seuil.

NøLKE Henning, 1983, "Les adverbes paradigmatisants ; fonction et analyse ", Revue Romane 23, Copenhague, Academisk Forlag. 
PHILIPPE Gilles, 2002, "L'appareil formel de l'effacement énonciatif et la pragmatique des textes sans locuteur " in Amossy, R., dir., Pragmatique et analyse des textes, 17-34. Université de Tel-Aviv.

PLANTIN Christian, 2002, article "Explication ", in Charaudeau P. et Maingueneau D., Dictionnaire d'Analyse du Discours, 251-254. Seuil, Paris.

RABATEL, Alain, 2003, "L'effacement énonciatif et ses effets pragmatiques de sous- et de sur-énonciation ", Estudios de Lengua y Literatura francesas 14, Université de Cadix, 33-61.

SIMONIN GRUMBACH Jenny, 1975, "Pour une typologie des discours ", in Kristeva J., Milner, J.-C., Ruwet, N., dir., Langue, discours, société : pour Émile Benveniste, 85-121. Seuil, Paris.

SWALES, J., 1990, Genre Analysis: English in academic and research settings, Cambridge University Press, Cambridge.

WILMET Marc, 1998, $2^{e}$ éd., Grammaire critique du français, Hachette-Duculot, Louvain-laNeuve. 